\title{
Contribution à l'étude de la biologie d'Asellus cavaticus Leydig
}

\author{
(Note préliminaire) \\ Par Jean-Paul Henry ${ }^{1}$ ) \\ Avec Planche \%0 (1)
}

La faune des eaux souterraines comprend un grand nombre de genres et d'espèces troglobies de Crustacés Isopodes appartenant à des familles variées (Asellides, Cirolanides, Sphéromides); les unes ont encore des formes alliées dans les eaux douces épigées (Asellus) alors que d'autres sont isolées et cantonnées dans le domaine souterrain (reliques comme Caecosphaeroma).

Une des espéces de cet ordre la plus anciennement connue comme cavernicole est l'Asellus cavaticus Leydig qui a été signalée dans presque toute l'Europe (Angleterre, Belgique, Allemagne, France, Suisse et Autriche, etc.). Malgré cette vaste répartition sa biologie est peu ou mal connue, aussi nous sommes nous donné comme but de recherches l'étude écologique et éthologique de cette espèce grâce à des élevages entrepris au laboratoire dans des conditions se rapprochant le plus possible de celles du biotope origine.

R. Husson et J. Daum avaient effectué, il y a quelques années des recherches expérimentales sur la biologie de ce cavernicole, mais les élevages durent être interrompus. C'est pourquoi Monsieur le Professeur Husson nous a fait reprendre systématiquement ces études sur une plus grande échelle et avec des individus provenant non plus d'une mais de deux stations.

Nos premières observations poursuivies pendant près de vingt mois viennent étayer les résultats entrevus précédemment par les deux auteurs cités ci-dessus et nous nous proposons ici de synthétiser les résultats obtenus.

\section{Récoltes}

Le matériel en élevage provient de deux stations: la mine de fer de Moutiers (Meurthe-et-Moselle) et la grotte de Sainte-Reine près de Toul (Meurthe-et-Moselle). Les résultats exposés dans cette note

1) Laboratoire de Biologie Animale et Générale Faculté des Sciences, 6 Bd Gabriel, Dijon, France. 
intéressent plus particulièrement les individus de la grotte de SainteReine, récoltés en premier et en plus grand nombre. Nous ferons d'ailleurs toujours la distinction entre les exemplaires des deux stations, car s'ils appartiennent à la même espèce casaticus, ils présentent des différences très notables de taille. Les Aselles de Moutiers peuvent atteindre $7,8 \mathrm{~mm}$ pour les femelles, $8,4 \mathrm{~mm}$ pour les mâles, tandis que ceux de Sainte-Reine ne dépassent pas $5,5 \mathrm{~mm}$ pour les femelles et 4,8 mm pour les mâles. Dans chacune de ces deux stations, la plus petite femelle ovigère mesure respectivement $7,3 \mathrm{~mm}$ et $3,4 \mathrm{~mm}$. Il semble bien que l'on doive envisager l'existence d'une grande et d'une petite forme et nous serons amenés ultérieurement à étudier l'incidence que peut avoir sur la biologie de ces deux populations cette remarquable différence de taille.

Au total, nous avons récolté 205 Aselles se répartissant ainsi:

Tableau 1

\begin{tabular}{l|c|c|c|c|c|c}
\hline $\begin{array}{c}\text { Récoltes } \\
\text { Dates }\end{array}$ & $\begin{array}{c}\text { Moutiers } \\
26.9 .61\end{array}$ & $\begin{array}{c}\text { Moutiers } \\
3.11 .61\end{array}$ & $\begin{array}{c}\text { Ste-Reine } \\
15.4 .61\end{array}$ & $\begin{array}{c}\text { Ste-Reine } \\
\mathbf{1 . 5 . 6 1}\end{array}$ & $\begin{array}{c}\text { Ste-Reine } \\
27.9 .61\end{array}$ & $\begin{array}{c}\text { Ste-Reine } \\
\mathbf{1 4 . 8 . 6 2}\end{array}$ \\
\hline Nombre de o & 3 & 17 & 17 & 1 & 11 & 20 \\
Nombre de + & 5 & $\mathbf{1 4}$ & $\mathbf{2 4}$ & $\mathbf{4}$ & 28 & 35 \\
Nombre de + & & & & & & \\
ovigères & 5 & 6 & 4 & 4 & 2 & 5 \\
Total & 13 & 37 & 45 & 9 & 41 & 60
\end{tabular}

\section{Biologie sexuelle}

a) Epoques d'incubation

La prédominance du nombre des femelles par rapport à celui des mâles qui avait déjà été constatée par R. Husson et J. Daum se traduit très nettement à la lecture du tableau ci-dessus, puisque pour 136 femelles capturées il n'y a que 69 mâles. Au cours de leurs récoltes (en octobre et décembre) effectuées à la mine de Moutiers, ces auteurs avaient dénombré sur 40 femelles récoltées 23 femelles ovigères ce qui est une proportion très importante et concluaient à la nécessité d'un élevage en laboratoire étalé sur plusieurs mois pour établir s'il existait une période de plus grande activité sexuelle. Nos élevages ne nous ont pas encore permis de préciser avec certitude le cycle sexuel chez les Aselles de Moutiers, par contre pour ceux de Sainte-Reine les résultats peuvent se traduire dans le diagramme et l'histogramme de la planche 1.

Ils permettent de constater:

- que pendant toute l'année, on peut trouver des femelles ovigères, 
- qu'il semble bien cependant qu'une période de plus grande activité sexuelle se manifeste entre février et mai, et que par la suite un ralentissement très net apparaît dans le nombre des femelles ovigères à partir du mois de juin.

Ces résultats établis sur un an et sur un nombre limité d'individus demandent à être confirmés sur un matériel plus important pendant une durée d'élevage plus longue. Néanmoins, il est très intéressant de comparer dès à présent ces premières observations avec celles que Ginet a faites sur l'Amphipode souterrain Niphargus virei Chevreux. Il note que la reproduction qui s'effectue toute l'année présente un maximum en mai-juin et un minimum en novembre-décembre. Ce léger retard du maximum peut s'expliquer facilement quand on sait que l'auteur détermine cette périodicité saisonnière des pontes par l'observation des femelles récoltées ovigères n'ayant obtenu dans ses élevages que 19 pontes seulement, dont la moitié d'ailleurs en marsavril. Nous pouvons donc noter qu'il y a chez ces deux espèces cavernicoles un remarquable synchronisme des époques de plus grande activité sexuelle.

\section{b) Durée d'incubation}

Dans nos élevages nous nous sommes plus particulièrement attachés à observer les femelles pour en rechercher les ovigères, ce qui nous a conduit à déterminer chez ces dernières leur durée d'incubation, qui était encore inconnue. Nos observations furent faites sur des femelles devenues ovigères au laboratoire. En effet en 1959, R. H u s s on signale qu'il obtient à diverses reprises des pontes parmi ses élevages. A notre tour, nous avons obtenu de nombreuses pontes à partir de femelles récoltées non ovigères et mises en élevage avec des mâles, bien que nous n'ayons encore pu observer aucun accouplement, qui reste donc encore inconnu.

Ginet signale la rareté du phénomène chez Niphargus par comparaison avec les Amphipodes épigés chez lesquels la copulation est facile à observer dans la nature ou en élevage; la même différence est à noter chez les Aselles dont l'accouplement des formes épigées est connu depuis longtemps. Par contre, on l'observe couramment chez un autre Isopode des eaux souterraines Caecosphaeroma burgundum Dollfus (Husson, 1953; D a um, 1954).

Dès qu'une femelle est reconnue être ovigère, elle est isolée dans un récipient et observée régulièrement jusqu'à la mise-bas. Nos observations qui ont porté sur plus de cinquante femelles de tailles différentes montrent que la durée d'incubation varie entre 2 mois 10 jours et 2 mois 20 jours. La moyenne de nos observations s'établissant à 2 mois 
17 jours entre la ponte des œufs dans le marsupium et la naissance des jeunes Aselles.

Cette durée de vie marsupiale est courte si on la compare à celle observée chez Caecosphaeroma burgundum (11 à 12 mois) ou chez Niphargus virei (4 mois 10 jours). Asellus cavaticus ne paraît donc pas présenter, en ce qui concerne son développement, la lenteur qui caractérise normalement le comportement des troglobies. Par ailleurs, Husson et Daum remarquaient que le rythme des mues des jeunes Aselles se rapprochait légèrement de celui des Aselles épigés. Nous avons entrepris d'étudier le cycle biologique d'Asellus meridianus Racovitza, Aselle épigé assez commun dans les eaux douces de notre région et appartenant au mème groupe qu'Asellus cavaticus. Nos recherches ne nous permettent pas encore de donner des résultats, mais nous nous proposons de vérifier cette idée souvent émise qu'Asellus cavaticus est un cavernicole récent dont l'immigration dans le domaine souterrain pourrait être due par exemple à un changement de climat.

\section{c) Mues post-incubatoires}

Peu de temps après s'être libérées les femelles effectuent une mue en deux temps. D'abord une mue postérieure qui comprend le pléotelson et les trois derniers péréionites, puis une mue antérieure du reste du corps. R. Huss on et J. D a um avaient constaté que la mue postérieure se produisait quelques jours après la naissance des jeunes et qu'elle était suivie de quelques heures seulement par la mue antérieure. Nos observations sur les femelles de la grotte de Sainte-Reine nous permettent de préciser ces résultats et de constater une légère différence avec les individus de Moutiers. Pour les exemplaires de la première station, la mue postérieure n'a jamais eu lieu avant le cinquième jour suivant la mise-bas, à deux exceptions près, et elle se produit toujours avant le onzième jour. L'exuviation antérieure se produit normalement 1 à 4 jours après; ce délai est réduit à quelques heures chez une seule femelle de cette station alors que c'est la règle pour celles de Moutiers.

\section{d) Nombre de jeunes}

Le grand nombre de femelles ovigères obtenues dans notre élevage nous a conduit à vérifier l'idée que la taille de la femelle est en rapport avec le nombre de jeunes. Nous donnons dans le tableau ci-dessous pour 12 femelles de taille décroissante le nombre de jeunes qu'elles ont libérés, la durée d'incubation et les temps de la mue post-incubatoire. 
Tableau 2

\begin{tabular}{c|c|c|c|c|c}
\hline $\begin{array}{c}\text { Femelles } \\
\text { ovigères }\end{array}$ & $\begin{array}{c}\text { Durée d'incu- } \\
\text { bation en jours }\end{array}$ & $\begin{array}{c}\text { Mue post. } \\
\text { en jours }\end{array}$ & $\begin{array}{c}\text { Mue ant. } \\
\text { en jours }\end{array}$ & $\begin{array}{c}\text { Taille } \\
\text { en mm }\end{array}$ & $\begin{array}{c}\text { Nombre } \\
\text { de jeunes }\end{array}$ \\
\hline 1 & 75 & 8 & 1 & 5,5 & $12(31)$ \\
2 & 71 & 8 & 1 & 5 & 12 \\
3 & 75 & 5 & 2 & 5 & 29 \\
4 & 70 & 9 & 2 & 4,8 & 21 \\
5 & 72 & 9 & 2 & 4,8 & $27(12)$ \\
6 & 71 & 5 & 2 & 4,4 & 23 \\
7 & 80 & 4 & 3 & 4,1 & 7 \\
8 & 71 & 5 & 2 & 4 & 10 \\
9 & 82 & 5 & 2 & 3,8 & 9 \\
10 & 70 & 10 & 4 & 3,7 & 7 \\
11 & 79 & 6 & 5 & 3,5 & 8 \\
12 & 76 & 4 & 3 & 3,4 & 7
\end{tabular}

Une première constatation s'impose: d'une part les femelles (sauf une) dont la taille est ou dépasse $4 \mathrm{~mm}$ ont donné naissance à dix jeunes au moins. C'est une de plus grandes femelles $(5 \mathrm{~mm})$ qui a obtenu le plus grand nombre de jeunes (29). D'autre part à une taille inférieure à $4 \mathrm{~mm}$ correspond un nombre de jeunes n'atteignant pas 10 . Ces résultats viennent en confirmation avec l'idée émise plus haut et généralement admise. Seulement, on voit que cette règle n'est pas absolue puisque deux femelles de même taille $(5 \mathrm{~mm})$ ont obtenu un nombre très inégal de jeunes (12 et 29); une femelle de $5,5 \mathrm{~mm}$, c'est-àdire la plus grande de notre élevage n'a libéré que 12 jeunes, ce qui est très faible pour sa taille. Mais cette même femelle, au cours d'une deuxième mise-bas a donné naissance cette fois à 31 jeunes. L'inverse pouvant se produire d'ailleurs; ainsi la femelle $\mathrm{n}^{\circ} 5$ de notre tableau qui a donné à la première mise-bas 27 jeunes n'en a libéré que 12 lors d'une seconde mise-bas.

Ajoutons que c'est une femelle récoltée ovigère mesurant $5,3 \mathrm{~mm}$ qui détient le record du plus grand nombre de jeunes, soit 46. Il faut signaler aussi que nous avons noté environ 10\% de femelles dont les œufs avortaient après la ponte.

Ces premiers résultats semblent nous indiquer que si la taille de la femelle est un facteur important dans la quantité de jeunes, il doit également en exister d'autres qui peuvent avoir une influence sur ce nombre. On peut même penser qu'il existe un cycle où se répéterait régulièrement un effectif de jeunes élevé suivi d'un plus faible. Des recherches sont en cours pour vérifier cette hypothèse. 


\section{Conclusion}

Ces indications préliminaires que nous venons d'exposer ne prétendent pas tout dire sur la biologie sexuelle d'Asellus cavaticus. Nous avons souligné d'ailleurs pour chacun des résultats la nécessité de nouvelles et plus longues observations.

Néanmoins certaines particularités biologiques de cette espèce troglobie sont maintenant suffisamment établies pour nous permettre de les comparer à celles d'une espèce souterraine américaine Asellus tridentatus Hungerford, qui fut étudiée et mise en élevage par H of f m a n $n$ (1933). Asellus tridentatus est un Aselle cavernicole qui vit dans les puits et les sources et qui présente un régime carnivore, se nourrissant de larves de Diptères et même de ses congénères morts. Au passage, on remarquera que nos Aselles mangent principalement du bois pourri et des feuilles mortes et ne montrèrent jamais d'affection pour les proies animales. Les femelles d'Asellus tridentatus semblent beaucoup moins nombreuses que les mâles, puisque Hoffmann n'a dénombré que 31 femelles sur 192 individus récoltés. Il existerait done une différence notable dans le sexe-ratio de ces deux espèces. Cette faible proportion de femelles semblerait indiquer qu'il s'agit là d'une espèce peu prolifique ainsi que d'autres observations sur sa biologie sexuelle viennent le confirmer. En effet, s'il est vrai que cet Aselle américain est de grande taille et que les femelles atteignant 10 à $14 \mathrm{~mm}$ peuvent donner 40 à 70 jeunes, aucune femelle ovigère ne fut trouvée de décembre à juin (H ung erford 1922). Nous n'avons jamais constaté dans nos récoltes et nos élevages un pareil cas de repos sexuel chez Asellus cavaticus. De plus le fait remarquable que les femelles s'étant libérées de leurs jeunes meurent quelques jours après la mue post-incubatoire semble venir appuyer notre idée que l'espèce américaine est moins prolifique que la nôtre; car chez cette dernière les femelles non seulement continuent à vivre après la mise-bas, mais en outre semblent avoir un cycle de ponte assez rapide, puisque depuis le début de nos élevages cinq femelles devinrent ovigères à deux reprises.

Il était intéressant de rapprocher ici ces premières observations sur Asellus cavaticus de celles d'une autre espèce cavernicole. Comme nous le voyons, la biologie de l'une n'est pas obligatoirement celle de l'autre, malgré le même biotope et la parenté systématique.

\section{RÉSUMÉ}

Un élevage de l'Aselle cavernicole Asellus casaticus Leydig entrepris depuis plus de vingt mois nous permet de préciser quelques points du cycle sexuel de cette espèce. Une périodicité saisonnière des pontes semble avoir 
lieu avec un maximum au printemps comme c'est le cas chez l'Amphipode souterrain Niphargus virei Chevreux. La durée moyenne de vie marsupiale qui apparaît beaucoup plus courte que chez d'autres espèces troglobies, telles que Niphargus virei Chevreux ou Caecosphaeroma burgundum Dollfus, rapproche le cycle biologique de notre espèce de celui de Aselles épigés. Le nombre important de femelles devenues ovigères dans nos élevages (52) nous permet de souligner aussi que l'effectif de jeunes paraît en rapport avec la taille de la femelle mais qu'il doit exister d'autres facteurs influençant celuici. La comparaison des mêmes observations chez un Aselle cavernicole américain Asellus tridentatus Hungerford nous montre que leur biologie sexuelle semble assez différente.

\section{SUMMARY}

The cavernicole asellid Asellus casaticus Leydig has been reared in our laboratory for more than twenty months, permitting us to give some data on the sexual cycle of this species. Females provided with brood pouches seem to be more numerous in the spring, as is the case with the subterranean amphipod Niphargus virei Chevreux. The average length of the incubation period seems much shorter than that of other troglobitic species such as Niphargus virei Chevreux or Caecosphaeroma burgundum Dollfus, so that the life cycle of our species is nearer to that of epigean Asellus. The number of young per brood appears to be related to the length of the female, as is suggested by our observations on 52 ovigerous females, but there must be other factors which influence this quantity. The comparison between our observations and those made on the North American cavernicole Asellus tridentatus Hungerford shows that the sexual biology of these two species is apparently quite different.

\section{B I B LIOGRAP H IE}

Chappuis, P. A. (1949) - Les Asellides d'Europe et pays limitrophes. Arch. Zool. exp. et géné. 86: 78-94.

DAum, J. (1954) - Zur Biologie einer Isopodenart unterirdischer Gewässer: Caecosphaeroma burgundum Dollfus. Ann. Univers. Sarav. 3: 104-160.

Ginet, R. (1960) - Ecologie, éthologie et biologie de Niphargus (Amphipodes Gammaridés hypogés). Ann. Spéléo. 15: 239-376.

Gravier, C. (1931) - La ponte et l'incubation chez les Crustacés. Ann. Sc. Nat. Zool. 14: 309-419.

Henry, J.-P., et Magniez, G. (1962) - Présence d'Asellus meridianus en Bourgogne. Hydrobiologia 19: 334-338.

Hoffmann, C. H. (1933) - The biology of Caecidotea tridentata Hungerford (Isopoda, Crustacea). Journ. Kansas Entom. Soc. 6: 26-33.

HUNGERFORD, H. B. (1922) - A new subterranean Isopod from Kansas. The Kansas Univers. Sc. Bull. 14: 175-181.

Husson, R. (1938) - La faune des galeries de mines de l'Est de la France. C.R. 1er Cong. Lorr. Sc. sav. Est Fr. 1-6.

- (1953) - Considérations sur la biologie des Crustacés cavernicoles aquatiques (Niphargus, Caecosphaeroma, Asellus). C.R. 1er Cong. Intern. Spéléo. Paris, 3: 65-70.

- (1959) - Les Crustacés péracarides des eaux souterraines; considérations sur la biologie de ces cavernicoles. Bull. Soc. Zool. Fr. 84: 219-231. 
Husson, R., et Daum, J. (1955) - Über Haltung und Biologie von Asellus casaticus Leydig. Ann. Univers. Saras. 4: 137-144.

Remy, P. (1948) - Sur quelques Crustacés cavernicoles d'Europe. Notes biosp. 3: 35-47.

EXPLICATIONS DE LA PLANCHE 70 (1)

En haut: Périodicité mensuelle des pontes: en hachuré les femelles ovigères, en pointillé les femelles non ovigères; le plus petit nombre se rapporte aux femelles ovigères, le plus grand au nombre total des femelles en élevage pendant le mois considéré. - En bas: Périodicité mensuelle des pontes: proportion relative des femelles ovigères par rapport à 100 femelles. 

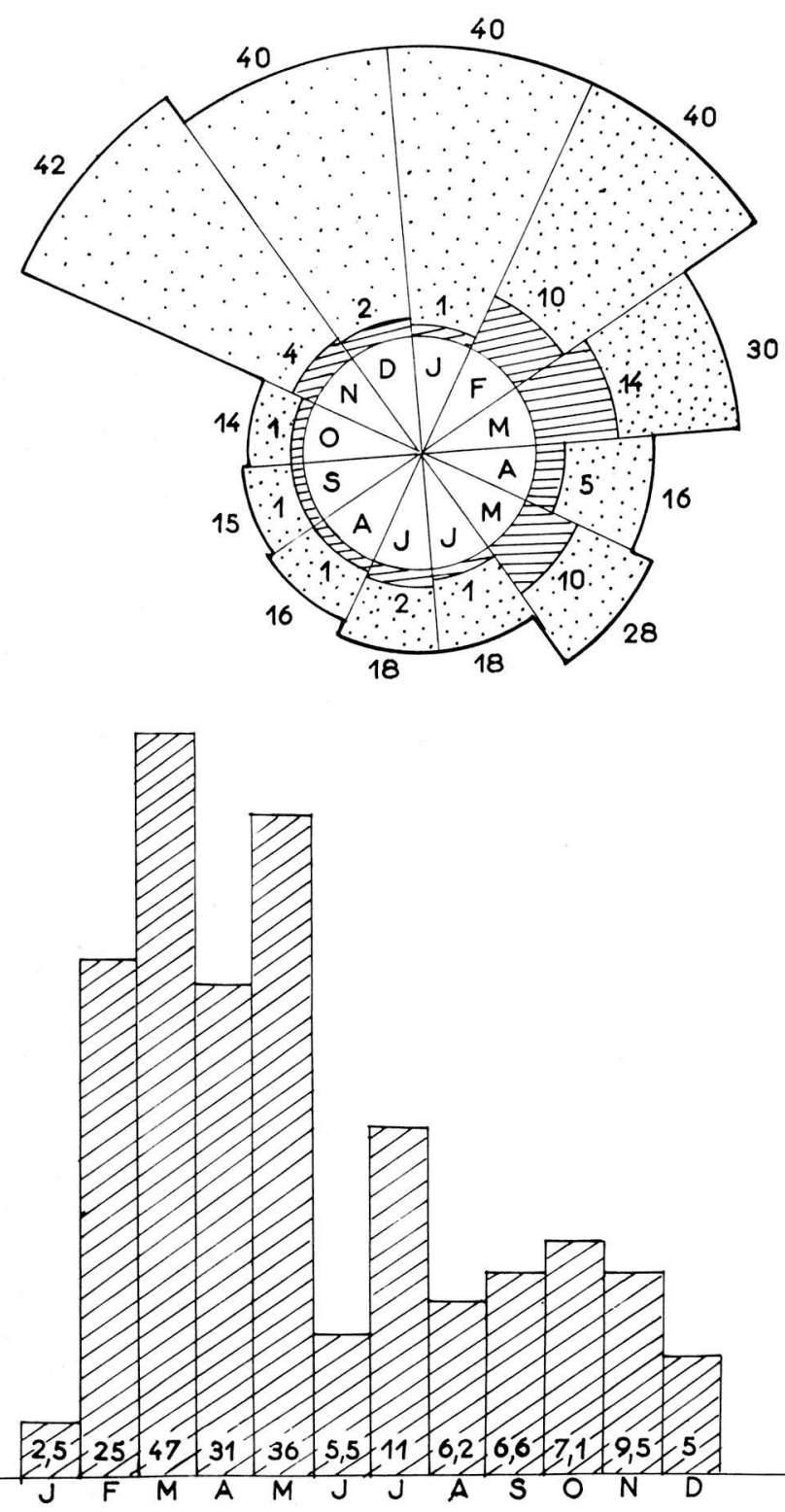